\title{
Assessment and Management of Pain in the Intensive Care Unit
}

\author{
Mohammad Ibrahim Aljumah ${ }^{1}$, Enass Farouk Aboshoushah ${ }^{2}$, Daniel Coric ${ }^{3}$, Alaa Matuq \\ Alaithan ${ }^{4}$, Abdullah Abdulmohsen Abdullah Almulhim ${ }^{5}$, Naif Matar AlOtaibi ${ }^{6}$, Mazen Hassan \\ Sadoun Alaslani ${ }^{7}$, Fahad hussain kammas ${ }^{8}$, Ayat Salman al saeed ${ }^{9}$, Ali breek alharthi ${ }^{10}$ \\ 1- Western Sydney University, 2-King Abdulaziz University Hospital, 3-Nepean Hospital, NSW, \\ Australia, 4- King Fahad Hospital Hofuf,5- ER resident in King Fahad Hospital Hofuf, 6- Prince \\ Sultan Military Medical City, 7- GP of Comprehensive Clinics of Ministry of Interior-Riyadh , 8- \\ King Fahad General Hospital Jeddah , 9- Alfaisal University , 10- King Abdullah Hospital . Bisha \\ city-ICU resident
}

\begin{abstract}
Background: Management and assessment of pain in patients in critical care unit is a daily challenge for the health care professionals, particularly in the intubated patients, patients on mechanical ventilation, or patients who are on analgesia as such patients are unable to self-assess existence and intensity of pain. It is crucial for physicians to follow guidelines that help in pain assessment and management effectively.

Aim of the work: Our aim in this study was to study how to evaluate and manage pain in an intensive care unit patient, predominantly those patients who are unable to self- report or assess.

Materials and methods: We conducted this review using a comprehensive search of MEDLINE, PubMed, and EMBASE, January 1994, through March 2017. The following search terms were used: pain in ICU, pain management in ICU, pain assessment methods, pain assessment in intubated patients.

Conclusion: Physical clues, which are given by comatose and intubated patients in critical care unit, should be taken in consideration, as they can be used as a method to identify existence of pain, and must be followed by effective management to decrease discomfort and prevent acute and chronic adverse effects.
\end{abstract}

Keywords: Pain in ICU, Intensive care unit assessment of pain, ICU pain management, guidelines to pain management.

\section{INTRODUCTION}

According to the International Association for Study of Pain (IASP), pain is the emotional and sensory unpleasant experience that is associated with the presence of potential (or actual) damage. From this previous definition, we can conclude that the nature of pain is subjective, meaning that the person who is feeling it can only assess its intensity. However, there are categories of patients who are not able to assess the intensity of their pain, or even report it ${ }^{[1]}$. These include, for example, patients who are intubated, patients who are on mechanical ventilation, patients who are admitted to the intensive care unit, and other patients. Therefore, the proper assessment of pain in intensive care unit patients is considered a challenge for clinicians. This challenge adds to other challenges faced when dealing with intensive care unit patients in general, like existing comorbidities, dementia, psychosis, delirium, along with other mental and neurological disorders ${ }^{[2]}$.

\section{METHODOLOGY}

\section{- Data Sources and Search terms}

We conducted this review using a comprehensive search of MEDLINE, PubMed, and EMBASE, January 1994, through March 2017. The following search terms were used: pain in ICU, pain management in ICU, pain assessment methods, pain assessment in intubated patients.

\section{- Data Extraction}

Two reviewers have independently reviewed the studies, abstracted data, and disagreements were resolved by consensus. Studies were evaluated for quality and a review protocol was followed throughout. The study was done 
after approval of ethical board of King Abdulaziz University.

\section{Types of Pain experienced by ICU patients}

There are many causes that can lead to the development of pain in patients at risk in critically ill patients. These include traumas, surgical procedures, malignancies, burns, and other causes. Therefore, a proper classification of pain is needed to help establish sufficient pain management plans. Generally, we classify pain into the following groups ${ }^{[3]}$ :

I. Pain that is continuous and associated with surgical procedures.

II. Ongoing disease pain that is acute.

III. Pain that is intermittent and can be associated with procedures in the intensive care unit.

IV. Pain that is chronic and had been present before the admission of the patients to the intensive care unit.

Other consequences of intensive care unit pain are the resulting compromises in oral cavity suction, bronchial tree suction, changing patients' positions, wound care, removal of drain, insertion of catheters, and establishing IV accesses. Some long-term consequences can result from this pain, most importantly, the prolonged resulting pain that will significantly decrease patients' quality of life after they are discharged from the intensive care unit. All these types of pain are significantly related to sex, age, and prior pain conception with previous interventions. Despite being that common, pain is properly managed only in about $25 \%$ of intensive care unit patients ${ }^{[4]}$.

\section{Incidence of pain in ICU patients}

A recent report published by the World Health Organization (WHO) estimated that the largest portion of world population (over 83\%) do not have access to proper, sufficient pain management, with the presence of cases with no pain management at all ${ }^{[5]}$. When it comes to patients in the intensive care unit, pain is considered to be common, which is well-documented. In fact, it was estimated that up to $70 \%$ of patients admitted to the intensive care unit, will suffer from moderate (or even severe) pain, at least once in the course of their admission. Another report estimated that pain at rest can be experienced in about $30 \%$ of intensive care unit patients, and pain with simple medical procedures can be experienced in about half intensive care unit patients ${ }^{[6]}$.

A large number of discharged patients who were admitted to the intensive care unit have reported that during their admission, they suffered from significant distress that was related to pain. Having altered consciousness levels in these patients or having any other cause that prevents them from verbal communication, led to the failure of proper pain assessment, and failure of achieving sufficient pain control, leading to this significant distress. Therefore, physicians should keep in mind that patients, who fail to verbally communicate, are not necessarily free of pain. These patients may require some kind of analgesics or pain management. Clinicians should always be aware that it is essential to properly, and continuously evaluate and assess pain in all patients who have problems with verbal communication ${ }^{[7]}$.

\section{Consequence of ICU Pain}

Improper pain management can lead to both psychological and physiological adverse events that could be chronic, and sometimes serious. Most patients, who were previously admitted to the intensive care unit, reported that they suffered from pain during their admission. This pain was the main cause of preventing them from getting sufficient sleep, even after their discharge. A recent report found that in about $82 \%$ of intensive care unit patients who suffered from pain, the cause of this pain was the endotracheal tubes. Moreover, this report stated that about $77 \%$ of patients suffered from moderate to severe pain. It was concluded that about $17 \%$ suffered from severe pain during their intensive care unit admission, which lasted for more than six months even after their discharge. Moreover, up to $18 \%$ of patients were found to have high likelihood of developing post-traumatic stress disorder because of the pain they suffered from ${ }^{[3,8]}$.

In some cases, this pain might cause the development of significant long-term consequences. Increase in the concentrations of epinephrine, nor-epinephrine and other catecholamines associated with pain will lead to vasoconstriction, significant declined in 
tissue perfusion, and dysfunctions of oxygen partial pressure regulation in organs.

Moreover, pain is associated with increased metabolic rate leading to protein catabolism, lipolysis, hyperglycemia, and dysfunctional healing of wounds, which will in turn lead to increased infections risk. In addition, pain has significant effects on the immune system. These effects include the inhibition of activity of natural killers, the decline in counts of cytotoxic T-lymphocytes, and the decrease of the neutrophils ability to perform phagocytosis. Neuropathic chronic pain can also develop in patients who experience severe acute pain ${ }^{[9]}$.

\section{Assessment of Pain in the ICU}

When managing critically ill patients, the first step in pain management is to properly assess this pain. It is essential to obtain reports measuring pain of these patients. However, although being very important, this approach is not enough in intensive care unit patients due to the common presence of communication dysfunctions in these patients. Endotracheal tubes, intake of analgesics, and severe diseases are all causes of inability to properly communicate in these patients. Therefore, it is important to assess other physiological, objective factors like blood pressure, heart rate, and respiration rate ${ }^{[10]}$. On the other hand, these physiological measures can sometime be biased by the underlying disease like hypoxia, hemorrhage, sepsis, or other disorders that may independently alter vital signs. In addition vital signs measurement is limited to only determining the presence or absence of pain, without describing its severity or other characteristics ${ }^{[2]}$.

Therefore, it is crucial to establish a proper toll to identify and assess pain in these patients. Several previous studies were not able to establish a strong correlation between blood pressure or heart rate and the presence of pain. Most procedures (either painless or painful) can affect vital signs and cause high blood pressure and/or tachycardia.

In conclusion, we could not base our assessment of pain on these measurements when dealing with patients in the intensive care unit ${ }^{[2]}$.

\section{Critical Care Pain Observation Tool (CPOT):}

The Critical Care Pain Observation Tool (CPOT) was primarily designed in French language. It was later officially translated and validated in other languages. The main goal of the Critical Care Pain Observation Tool (CPOT) was to provide an accurate perception of pain in patients who are critically ill, including patients in the intensive care unit. This tool includes four main categories ${ }^{[11,12]}$

-Facial expressions.

-Body movements.

-Muscle tension.

- Compliance with a ventilator in case of an intubated patient, and verbalization in case of an extubated patient.

Each of these categories has a score of zero, 1, or two, with a total score ranging between zero and 8. Data suggested that a score of 2 or less can be used to rule out the presence of significant pain, and a score higher than 2 can confirm the presence of pain in the patient [table 1] ${ }^{[11]}$.

\section{Behavioral Pain Scale (BPS):}

Payen et al. ${ }^{(13)}$ primarily designed the behavioral pain scale (BPS) as an approach to assess pain in unconscious patients and mechanically ventilated patients. This scale depended on the measurement of three behaviors ${ }^{[10,13]}$ :

1) facial expression,

2) Movements of the upper extremities and

3) compliance with a ventilator.

Both the family and the physician could use this score easily and assess the severity of pain in the patient. The behavioral pain scale has a total score between 3 and 12. A score of 3 is used to rule out the presence of pain, and a score of 12 is used to confirm the presence of severe pain (table 2) ${ }^{[13]}$. 
Table (1): Critical Care Pain Observation Tool (CPOT)

\begin{tabular}{|c|c|c|c|}
\hline Indicator & Description & Interpretation & score \\
\hline \multirow[t]{3}{*}{ Facial expression } & - No muscle observed & - $\quad$ Relaxed & 0 \\
\hline & $\begin{array}{l}\text { - Frowning, brow lowering, } \\
\text { orbit tightening, other } \\
\text { changes (eye opening, } \\
\text { tearing) }\end{array}$ & - $\quad$ Tense & 1 \\
\hline & 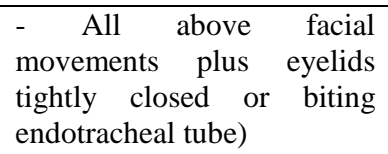 & - $\quad$ Grimacing & 2 \\
\hline \multirow[t]{3}{*}{ Body movements } & $\begin{array}{l}\text { - No movement or normal } \\
\text { position }\end{array}$ & - Relaxed & 0 \\
\hline & $\begin{array}{l}\text { - Slow, cautious movement, } \\
\text { attempting to touch pain } \\
\text { site, attention seeking } \\
\text { movements }\end{array}$ & - Protection & 1 \\
\hline & $\begin{array}{l}\text { - Pulling tube, attempting to } \\
\text { sit-up, striking staff, trying } \\
\text { to climb out of bed }\end{array}$ & - Agitation & 2 \\
\hline \multirow[t]{3}{*}{ Muscle tension } & $\begin{array}{l}\text { - No resistance to passive } \\
\text { movements }\end{array}$ & - Relaxed & 0 \\
\hline & $\begin{array}{l}\text { - Resistance to passive } \\
\text { movements }\end{array}$ & - Tense, rigid & 1 \\
\hline & - Strong resistance & -Very tense or rigid & 2 \\
\hline \multirow{3}{*}{$\begin{array}{l}\text { Compliance } \\
\text { ventilator } \\
\text { or } \\
\text { vocalization }\end{array}$} & $\begin{array}{l}\text { - Easy ventilation, no active } \\
\text { alarms }\end{array}$ & -Tolerating ventilator & 0 \\
\hline & $\begin{array}{l}\text { - Alarms may be } \begin{array}{r}\text { active, } \\
\text { coughing but } \\
\text { spontaneously }\end{array} \\
\end{array}$ & $\begin{array}{l}\text { - Coughing but tolerating } \\
\text { ventilator }\end{array}$ & 1 \\
\hline & $\begin{array}{l}\text { - Sighing, moaning, crying } \\
\text { out }\end{array}$ & - Fighting ventilator & 2 \\
\hline Total & & & $0-8$ \\
\hline
\end{tabular}

Table (2): Behavioral Pain Scale (BPS)

\begin{tabular}{|l|l|l|}
\hline Item & Description & Score \\
\hline Facial expression & - Relaxed & 1 \\
& - Partially tightened & 2 \\
& - Fully tightened & 3 \\
& - Grimace & 4 \\
\hline Upper limb movements & - No movement & 1 \\
& - Partially bent & 2 \\
& - Fully bent with finger & 3 \\
& flexion & 4 \\
\hline Compliance with mechanical & - Permanently retracted & - Tolerating movement \\
ventilation & - Coughing but tolerating & 1 \\
& - Fighting ventilator & 2 \\
& - Unable to control ventilation & 3 \\
\hline
\end{tabular}

\section{Management of Pain}

When dealing with critically ill patients in the intensive care unit, pain management should depend on continuous measurements and monitoring of pain in these patients, and planning potential interventions accordingly. Pain management mainly depend on self-assessment done by the patients themselves. Therefore, self-assessment should always be considered in all patients. Patients themselves mainly determine the intensity and level of pain. The visual analogue scale (VAS) is considered to be one of the best methods to self-assess pain ${ }^{[14]}$. The Numeric Rating Scale (NRS) on the other hand assumes that pain determination should depend on collaborating 
efforts from both the patient and the physician [15].

Sedation, delirium, agents used in delirium treatment, along with other factors, can all play an important role in the functioning of the central nervous system. It is essential, however, to keep in mind that all these tools may not be ideal in some subgroups and populations. These populations include patients with dementia, children, patients who have problems with verbal communication, and patients with any other consciousness disorder. This puts a relatively high proportion of patients under the risk of underassessment of their pain with normal self-assessment tools. To manage these populations properly, newer measures were formulated to be used. These depend on several factors, and mainly clinical observation by all the team responsible for care ${ }^{[12]}$.

In their latest guidelines, the Society of Critical Care Management (SCCM) did investigate six scales used to assess pain in patients with delirium and other significant causes of dysfunctional communication. These scales were: Non-verbal Pain Scale (NVPS), Critical Care Pain Observation Tool (CPOT), BPS-non-intubated, the Pain assessment Intervention, Notation (PAIN) algorithm, and the Pain Behavioral Assessment Tool (PBAT). Authors concluded that the best scales of these, and the one associated with better and more accurate results in patients with dysfunctional verbal communication were the BPS and the Critical Care Pain Observation Tool (CPOT) ${ }^{[11]}$.

Another important factor in the management of pain in intensive care unit patients is the family. Family members have been found to significantly help in achieving proper assessment of pain in patients admitted to the intensive care unit. This is due to their ability to faster detect changes in patients' behavior and relate these changes with pain. An advantage of both the BPS and the Critical Care Pain Observation Tool (CPOT), is their ability to be used easily by family members [16].

\section{Medications Used to Treat Pain in the ICU}

When dealing with critically ill patients in the intensive care unit, the most important medications that are used for pain management are opioids. Used opioids include remifentanil, methadone, hydromorphone, fentanyl, and morphine. The selection of the proper opioid and dosing depends on several factors, along with the drug's characteristics. Meperidine is not usually used in patients in the intensive care unit due to its potential related neurotoxicity. Other than opioids, some pain control drugs and analgesics can be used. These include non-steroidal anti-inflammatory drugs (NSAIDs) like ibuprofen and ketorolac, local anesthetics (bupivacaine), anticonvulsant, and intravenous acetaminophen. These alternatives are mainly used to decrease the necessity of high opioid doses and thus decrease the risk of dependence and possible abuse ${ }^{[17]}$

The use of acetaminophen intravenously for pain management was recently approved in the United States, after several studies indicating its efficacy with opioids, and relative safety. Its effects have been mostly established to treat intensive care unit patients who are having post-operative pain after cardiac surgery or other major surgeries ${ }^{[18]}$.

Neuropathic pain is a significant cause of poor quality of life in patients who are admitted to the intensive care unit. Unfortunately, neuropathic pain is sometimes hard to control even with the use of opioids. In such cases, carbamazepine and gabapentin are administrated to intensive care unit patients. However, caution should be made when giving this treatment, making sure that gastrointestinal functions of patients are well functioning. Patients who have gastrointestinal dysfunctions may have difficulties with these pain management medications, leading to limited efficacy ${ }^{[19]}$.

In some cases of severe post-operative pain following major or multiple procedures, neuraxial analgesia may be used. Other possible interventions for pain management are non-pharmacological and include massage, music therapy, and relaxation techniques. These may play a role in decreasing the pain and in turn the need for high doses of pharmacological agents. Moreover, they are safe, cheap, and readily available ${ }^{[20]}$.

\section{CONCLUSION}

Critically ill patients admitted to the intensive care unit commonly suffer from pain that can range from mild to extremely severe. This pain could be attributed to comorbidities, previous procedures, and other etiologies. As most patients in the intensive care unit are critically ill, they are unable to verbally 
communicate and report pain they are experiencing. Therefore, physical signs in these patients must always be monitored to detect and assess pain. Recent guidelines for pain management should be well applied by physicians, nurses, and all the team responsible for the care of the patient. Rapid and effective relief of pain play a significant role in decreasing stress, preventing complications and improving quality of life after discharge.

Refrences

1. Treede R D (2018): The International Association for the Study of Pain definition of pain: as valid in 2018 as in 1979, but in need of regularly updated footnotes. Pain Rep., 3: e643.

2. Puntillo K A, Neuhaus J, Arai S, Paul S M, Gropper M A, Cohen N H et al. (2012): Challenge of assessing symptoms in seriously ill intensive care unit patients: can proxy reporters help? Crit Care Med., 40: 2760-7.

3. Ayasrah S M, O'Neill T M, Abdalrahim M S, Sutary M M, Kharabsheh M S (2014): Pain Assessment and Management in Critically ill Intubated Patients in Jordan: A Prospective Study. Int J Health Sci (Qassim), 8: 287-98.

4. Lin R J, Reid M C, Liu L L, Chused A E, Evans A T (2015): The Barriers to High-Quality Inpatient Pain Management: A Qualitative Study. Am J Hosp Palliat Care, 32: 594-9.

5. Manjiani D, Paul D B, Kunnumpurath S, Kaye A D, Vadivelu N (2014): Availability and utilization of opioids for pain management: global issues. Ochsner J., 14: 208-15.

6. Gupta M, Sahi M S, Bhargava A K, Talwar V A (2016): Prospective Evaluation of Symptom Prevalence and Overall Symptom Burden Among Cohort of Critically Ill Cancer Patients. Indian J Palliat Care, 22: 118-24.

7. Stark L, Tofthagen C, Visovsky C, McMillan S C (2012): The Symptom Experience of Patients with Cancer. J Hosp Palliat Nurs, 14: 61-70.

8. Al Sutari M M, Abdalrahim M S, Hamdan-Mansour A M, Ayasrah S M (2014): Pain among mechanically ventilated patients in critical care units. J Res Med Sci., 19: 726-32.

9. Georgiou E, Hadjibalassi M, Lambrinou E, Andreou P, Papathanassoglou E D (2015): The Impact of Pain Assessment on Critically Ill Patients' Outcomes: A Systematic Review. Biomed Res Int., http://dx.doi.org/10.1155/2015/503830

10. Kotfis $\mathbf{K}$, Zegan-Baranska $\mathbf{M}$, Szydlowski L, Zukowski M, Ely E W (2017): Methods of pain assessment in adult intensive care unit patients - Polish version of the CPOT (Critical Care Pain Observation Tool) and BPS (Behavioral Pain Scale). Anaesthesiol Intensive Ther., 49: 6672.

11. Rijkenberg S, van der Voort $\mathbf{P} \mathbf{H}$ (2016): Can the critical-care pain observation tool (CPOT) be used to assess pain in delirious ICU patients? J Thorac Dis., 8: 285-7.

12. Severgnini P, Pelosi P, Contino E, Serafinelli E, Novario R, Chiaranda $M$ (2016): Accuracy of Critical Care Pain Observation Tool and Behavioral Pain Scale to assess pain in critically ill conscious and unconscious patients: prospective, observational study. J Intensive Care, 4: 68.

13. Payen J F, Bru O, Bosson J L, Lagrasta A, Novel E, Deschaux I et al. (2001): Assessing pain in critically ill sedated patients by using a behavioral pain scale. Crit Care Med., 29: 2258-63.

14. Haefeli M, Elfering A (2006): Pain assessment. Eur Spine J., 15 (1): 1724.

15. Tandon M, Singh A, Saluja V, Dhankhar M, Pandey C K, Jain P (2016): Validation of a New "Objective Pain Score" Vs. "Numeric Rating Scale" For the Evaluation of Acute Pain: A Comparative Study. Anesth Pain Med., doi: 10.5812/aapm.32101.

16. Khan $\mathbf{Z} \mathbf{H}$ (2013): Pain management in intensive care unit patients: the role of ultra-short acting opioid: remifentanil. Anesth Pain Med., 3: 188-90.

17. Gommers D, Bakker J (2008): Medications for analgesia and sedation in the intensive care unit: an overview. Crit Care, 12 (3): S4. 
18. Barr J, Fraser G L, Puntillo K, Ely E W, Gelinas C, Dasta J F et al. (2013): Clinical practice guidelines for the management of pain, agitation, and delirium in adult patients in the intensive care unit. Crit Care Med., 41: 263-306.

19. Gilron I, Watson C P, Cahill C M, Moulin D E (2016): Neuropathic pain: a practical guide for the clinician. CMAJ., 175: 265-75.

20. Barry D T, Savant J D, Beitel M, Cutter C J, Moore B A, Schottenfeld R S et al. (2012): Use of conventional, complementary, and alternative treatments for pain among individuals seeking primary care treatment with buprenorphine-naloxone. J Addict Med., 6: 274-9. 